\title{
Notwendigkeit, Sterbefasten differenzierter zu betrachten
}

\author{
Sabrina Fehn ${ }^{a}$, André Fringer ${ }^{b}$ \\ ${ }^{a}$ MSc; ${ }^{b}$ Prof. Dr., MScN (RN), beide: University of Applied Sciences St. Gallen, Institute of Applied Nursing Science
}

Die Aufnahme von Nahrung und Flüssigkeit ist ein höchst soziales Phänomen [1-3]. Aus diesem Grund kommt dieser Thematik auch im Umgang mit Patientinnen und Patienten hohe Bedeutung zu. Im Rahmen von Palliative Care ist dieses Phänomen bereits vielfältig erforscht und wurde sukzessive ein professioneller Umgang entwickelt [4-7]. Dennoch gibt es Wissenslücken, beispielsweise in Bezug auf den freiwilligen Verzicht auf Nahrung und Flüssigkeit [8]. Unter dem Schlagwort «Sterbefasten» erfährt dieses Thema derzeit breite Aufmerksamkeit.

«Freiwilliger Verzicht auf Nahrung und Flüssigkeit» (FVNF) ist eindeutig abgrenzbar gegenüber Nahrungsverweigerung sowie Anorexie und Kachexie. Zu FVNF liegt noch wenig Forschung vor. In der aktuellen wissenschaftlichen Aufarbeitung fällt auf, dass sich das Thema FVNF viel facettenreicher darstellt, als bisher angenommen. Der vorliegende Artikel hat zum Ziel, vier verschiedene Formen des freiwilligen Verzichts auf Nahrung und Flüssigkeit darzustellen.

\section{Dimensionen und Formen des FVNF}

In der Analyse aktueller Situationen, die uns von betroffenen Angehörigen berichtet wurden, konnten wir vier Formen des FVNF identifizieren. Für unsere For-

\section{Résumé}

Le refus d'alimentation et d'hydratation, parfois aussi appelé anorexie finale, revêt une importance croissante dans le domaine de l'autodétermination en fin de vie. Les connaissances actuelles mettent clairement en évidence un besoin élevé de recherche fondamentale qualitative et quantitative. Nos résultats d'étude en matière de refus d'alimentation et d'hydratation montrent que le phénomène est plus différencié qu'on ne le supposait jusqu'à présent. La manière de communiquer, en particulier, joue un rôle décisif. La personne a-t-elle expressément fait part de son refus ou l'applique-t-elle simplement sans que le sujet soit abordé? Cet aspect et d'autres témoignent du fait que le refus d'alimentation et d'hydratation ou la notion d'anorexie finale ne peuvent pas être utilisés en tant qu'étiquettes génériques, car le phénomène recouvre des réalités plus diverses que ces termes ne l'expriment. Cet article en décrit quatre formes. schungsarbeit hat sich zur Klassifizierung folgendes Notationssystem bewährt, wobei jeweils ein Buchstabe des Akronyms «FVNF» in Klammer stehen kann:

- FVNF: Freiwilliger Verzicht auf Nahrung und Flüssigkeit

- (F)VNF: (Freiwilliger) Verzicht auf Nahrung und Flüssigkeit

- FV(N)F: Freiwilliger Verzicht auf (Nahrung und) Flüssigkeit

- FVN(F): Freiwilliger Verzicht auf Nahrung (und Flüssigkeit)

Wie die Darstellung zeigt, lassen sich zwei Dimensionen unterscheiden: die Freiwilligkeit sowie die Art des Verzichts. Im Folgenden gehen wir auf die vier spezifischen Formen des FVNF näher ein.

\section{Die explizite Form des Verzichts: FVNF}

Die bewusste Entscheidung, auf Nahrung und Flüssigkeit zu verzichten, um das Leben vorzeitig zu beenden und das Sterben zu beschleunigen, stellt die bekannteste Form des FVNF dar und ist auch bekannt unter dem Namen «Sterbefasten». Hierbei handelt es sich um entscheidungsfähige Personen ohne psychische oder kognitive Beeinträchtigungen, die FVNF absichtlich wählen und dies offen kommunizieren [8-10]. Aus Fallberichten geht hervor, dass der Tod auf FVNF zurückzuführen ist, wenn er innerhalb von ein bis drei Wochen eintritt [11, 12]. Dies setzt voraus, den Verzicht vollständig und konsequent umzusetzen. Autonomie und Selbständigkeit bleiben fast bis zuletzt erhalten. Jedoch wird die Person in den letzten Lebenstagen zunehmend pflegebedürftig und benötigt professionelle 
Unterstützung $[13,14]$. Dann ist Palliative Care empfehlenswert, um Lebensqualität bis zuletzt zu sichern. Ab dem Zeitpunkt, an dem irreversible Beeinträchtigungen eintreten, sollte eine anwaltschaftliche Weiterführung der autonomen Entscheidung für FVNF erfolgen. Dies ging aus unseren Fallberichten hervor.

\section{Es bestehen kontroverse Diskussionen,} ob es sich bei dieser Form von FVNF um Suizid oder einen natürlichen Tod handelt.

Diese explizite Form des FVNF haben wir eher bei jüngeren Menschen beobachtet. Die jüngste Person war 48 Jahre alt. Die Angehörigen sind bei dieser Variante unmittelbar mit der Entscheidung der durchführenden Person konfrontiert und intensiv in das Geschehen involviert. Es bestehen kontroverse Diskussionen, ob es sich bei dieser Form von FVNF um Suizid oder einen natürlichen Tod handelt. In einschlägiger Literatur [15] zum Thema nimmt diese Variante breiten Raum ein.

\section{Die implizite Form des Verzichts: (F)VNF}

Die eher unbekannte Form des FVNF ist die nicht eindeutig kommunizierte, oft stillschweigende Reduktion der Nahrungs- und Flüssigkeitsaufnahme oder das Unvermögen, Nahrung und Flüssigkeit zu sich zu nehmen. Aus diesem Grund ist der Begriff «freiwillig» im Akronym eingeklammert (F)VNF. In unseren Untersuchungen kommt diese Form häufig bei hochaltrigen Menschen oder Personen in der Terminal-bzw. Sterbephase vor. Sie bringen ihre Entscheidung nicht explizit zum Ausdruck. Häufig ist dies darin begründet, dass sie zwar diesen Weg für sich gewählt haben, ihre Entscheidung jedoch nicht kommunizieren möchten oder nicht können. Diese Variante ist weniger Ausdruck eines persönlichen Willens zu Autonomie und Selbstgestaltung, sondern lässt sich eher als Folge von Lebensmüdigkeit verstehen.

Hier kann von einem natürlichen Verlauf die Rede sein, da das Verlangen nach Nahrung und Flüssigkeit abnimmt. Auf aussenstehende Personen wirkt (F)VNF als etwas Natürliches und nicht als Suizid. Bezüglich der Dauer gehen wir von einer ähnlichen Geschwindigkeit aus wie bei FVNF.

In Bezug auf (F)VNF konnten wir feststellen, dass von professioneller Seite die Gefahr besteht, betroffenen Personen pauschalisierend zu begegnen und sie zu etikettieren. Dies geschieht jedoch unreflektiert, was ein Problem darstellt. Im Rahmen von Videoanalysen der Nahrungsaufnahme von Menschen mit Demenz im Endstadium der Erkrankung konnten wir feststellen, dass Fachpersonen Nahrung und Flüssigkeit unter- schiedlich anreichen in Abhängigkeit von folgenden Faktoren:

- Fort- und Weiterbildung der Pflegefachpersonen

- Verständnis für einflusshemmende Strukturen und Rahmenbedingungen

- Körperspannungen der Betroffenen, die eine Nahrungsaufnahme verhindern.

In einem uns vorliegenden Fall wählte eine Heimbewohnerin (F)VNF und teilte dies erst während des Verlaufs ihrer Tochter mit. Den Pflegefachpersonen war dieser Entschluss bis zuletzt nicht bekannt. Dies macht deutlich, dass Fachpersonen einen nicht kommunizierten Verzicht als natürlichen Sterbeprozess interpretieren.

\section{Die stigmatisierte und verheimlichte Form: FV(N)F}

Eine bisher unbekannte Variante zeigte sich bei einer jüngeren, palliativ betreuten Patientin mit einer onkologischen Erkrankung. Ihr persönliches Umfeld übte so starken Druck auf sie aus, dass sie sich entschied, nicht mehr zu trinken. Nahrung jedoch nahm sie weiterhin zu sich, um die Angehörigen in Sicherheit zu wiegen. Für das familiäre Umfeld der Patientin stellte FVNF aus spirituellen Gründen keine Option dar, obwohl ein tiefes Bedürfnis der Patientin bestand. Zu Beginn kommunizierte die Patientin ihren Wunsch offen Ihre Entscheidungskompetenz war unbestreitbar. Die Familie sprach ihr jedoch aus spirituellen Gründen diese Entscheidungsfähigkeit ab. Dies traf auch auf das nachbarschaftliche Umfeld und den Seelsorger der ört-

\section{Den Pflegefachpersonen war dieser Entschluss} bis zuletzt nicht bekannt.

lichen Kirchengemeinde zu. Medizinische und pflegerische Palliative-Care-Fachpersonen gingen ebenfalls nicht auf den Wunsch der Betroffenen nach FVNF ein, sondern beugten sich dem Druck der Angehörigen [16]. Daraufhin entschied sich die Patientin für FV(N)F, hielt dies jedoch geheim und bezog lediglich eine enge Vertraute der Familie ein. Die Dauer des FV(N)F betrug ungefähr sechs Wochen. Was für das Umfeld der Betroffenen nach Suizid aussah, war für die Patientin selbst stimmig und etwas Natürliches [17].

\section{Ein natürlicher Prozess: FVN(F)}

Eine bekanntere Form des FVNF besteht im Verzicht auf Nahrung FVN(F), wie er beispielsweise in den letzten Lebenswochen oder -tagen natürlicherweise auftreten kann [18]. Appetitverlust stellt im Palliative- 
Care-Bereich ein bekanntes Phänomen dar. In der Terminalphase kommt es überwiegend vor, dass Betroffene keine Nahrung und Flüssigkeit mehr zu sich nehmen. Häufig besteht auf Seiten der Angehörigen die Angst, die betroffene Person könne verdursten und verhungern. Deshalb kann es vorkommen, dass - entgegen guter palliativer Praxis - nicht nur eine Flüssigkeitsgabe erfolgt, sondern auch die Verabreichung hochkalorischer Flüssignahrung, um die Angehörigen zu beruhigen. Die Dauer des FVN(F) ist erkrankungsabhängig. Diese Form des Verzichts tritt altersunabhängig auf.

\section{Die Einflussnahme der Angehörigen}

Anhand der vier Formen des FVNF wird deutlich, dass Angehörige eine wichtige Rolle im Umgang mit FVNF spielen. Unsere Untersuchungen zeigen, dass Angehörige Einfluss auf die Entscheidung der betroffenen Person zu nehmen versuchen und somit auch einen wichtigen Faktor bei der Durchführung des FVNF darstellen. Aus der Literatur ist bekannt, dass Angehörige bezüglich der Nahrungsaufnahme am Lebensende entweder

\section{Unsere Untersuchungen zeigen, dass Ange- hörige Einfluss auf die Entscheidung der betroffenen Person zu nehmen versuchen.}

der Gruppe der «Zustimmenden», «Schwankenden» oder "Ablehnenden» zuzuordnen sind. Trotz des Verständnisses der persönlichen Situation steht immer die Frage im Raum, ob man etwas unterlassen hat [3]. Somit ist es wichtig, dass Fachpersonen das Gespräch mit den Angehörigen suchen und sie über die jeweilige Form des FVNF aufklären [16].

\section{FVNF professionell begleiten}

Der vorliegende Beitrag stellt erstmals vier spezifische Formen des FVNF dar. Diese Differenzierung weist darauf hin, dass es nicht haltbar ist, freiwilligen Verzicht auf Nahrung und Flüssigkeit pauschal mit dem Etikett «Sterbefasten» zu versehen. Um mit der Entscheidung von Betroffenen für FVNF professionell umzugehen, ist es von zentraler Bedeutung, zunächst eindeutige Definitionen der vier spezifischen Formen zu entwickeln. Wichtig ist auch, Fachpersonen in Bezug auf die Kommunikation bei expliziten, vor allem aber bei impliziten Formen des FVNF zu sensibilisieren.
Disclosure statement

Die Autoren erklären, dass kein Interessenskonflikt besteht.

Literatur

1 Möhr A and Fringer A. Appetitlosigkeit - warum das Verlangen nach Essen nachlässt. pflegen: palliativ, 2013;17:1-8-11.

2 Wallin V, Carlander I, Sandman PO, Ternestedt BM, and Håkanson C. Maintaining ordinariness around food: partners' experiences of everyday life with a dying person. Journal of Clinical Nursing, 2014;23.10.1111/jocn.12518.

3 Johansson AE and Johansson U. Relatives' experiences of family members' eating difficulties. Scand J Occup Ther, 2009;16:1-2532.10.1080/11038120802257195.

4 Strasser F. Personalized, patient-centred cancer cachexia management: lessons to be learnt from oncology and to be taught to oncology. Current opinion in supportive and palliative care, 2013;7:4-342-344.10.1097/SPC.0000000000000018.

5 Oberholzer R, Blum D and Strasser F. The Concept of CachexiaRelated Suffering (CRS) in Palliative Cancer Care, in Diet and Nutrition in Palliative Care, V.R. Preedy, Editor. 2011, CRC Press Miami. p. 464.

6 Orrevall Y. Nutritional support at the end of life. Nutrition, 2015;31:4-615-6.10.1016/j.nut.2014.12.004.

7 Green SM and Watson R. Nutritional screening and assessment tools for older adults: literature review. Journal of Advanced Nursing, 2006;54:4-477-490.

8 Ivanović N, Büche D and Fringer A. Voluntary stopping of eating and drinking at the end of life - a 'systematic search and review' giving insight into an option of hastening death in capacitated adults at the end of life. BMC palliative care, 2014;13:1-1.10.1186/ 1472-684X-13-1.

9 Klein Remane U and Fringer A. Voluntary refusal of food and fluid in palliative care: a mapping literature review. Pflege, 2013;26:6-411-420.10.1024/1012-5302/a000329.

10 Black K and Csikai EL. Dying in the age of choice. Journal of Social Work in End of Life \& Palliative Care, 2015;11:1-27-49.

11 Bolt EE, Hagens M, Willems D and Onwuteaka-Philipsen BD. Primary care patients hastening death by voluntarily stopping eating and drinking. Annals of family medicine, 2015;13:5-421-428. 10.1370/afm.1814.

12 Chabot BE. Hastening death through voluntary cessation of eating and drinking: A survey, in Physician-Assisted Death in Perspective, S.J. Youngner and G.K. Kimsma, Editors. 2012, Cambridge University Press: Cambridge. p. 305-15.

13 Bickhardt J and Hanke RM. Freiwilliger Verzicht auf Nahrung und Flüssigkeit: Eine ganz eigene Handlungsweise. Deutsches Ärzteblatt, 2014;111:14-590-592.

14 Lachman VD. Voluntary stopping of eating and drinking: An ethical alternative to physician-assisted suicide. Medsurg nursing: official journal of the Academy of Medical-Surgical Nurses, 2015;24:1-56-59.

15 Chabot BE and Walther C. Ausweg am Lebensende: SterbefastenSelbstbestimmtes Sterben durch freiwilligen Verzicht auf Essen und Trinken. 4. Aufl. ed. München: Ernst Reinhardt Verlag; 2015

16 Del Rio MI, Shand B, Bonati P, Palma A, Maldonado A et al. Hydration and nutrition at the end of life: a systematic review of emotional impact, perceptions, and decision-making among patients, family, and health care staff. Psychooncology, 2012;21:9-913-21.10.1002/pon.2099.

17 McClement S. Cancer anorexia-cachexia syndrome: psychological effect on the patient and family. J Wound Ostomy Continence Nurs, 2005;32:4-264-268.

18 Berry EM and Marcus E-L. Disorders of Eating in the Elderly. Journal of Adult Development, 2000;7:2-87-99.10.1023/a:1009575706990. 